\title{
Endoparásitos en guanaco (Lama guanicoe). Revisión de situa- ción en Argentina y registros de la provincia de San Juan
}

\author{
Endoparasites in guanaco (Lama guanicoe). Review of the situation in Argentina \\ and registries in the province of San Juan
}

\author{
Cynthia Jesica Gonzalez-Rivas ${ }^{1,3}$, Carlos Eduardo Borghi ${ }^{1}$, Daniel Alfedro De Lamo ${ }^{2}$
}

\section{Resumen}

\begin{abstract}
Este estudio recopila los registros de especies endoparasitarias en guanacos (Lama guanicoe) de Argentina. Se hizo una revisión bibliográfica de los trabajos científicos publicados entre 1967 y 2017. Además, se incorporaron nuevos registros inéditos de localidades dentro de la provincia de San Juan. La fauna de endoparásitos estuvo representada por los phylum Nematoda, Platyhelminthes y Aplicomplexa, con un total de 65 registros totales, mencionando 26 especies y otras 14 que sólo fueron identificadas a nivel de género. Los nematodos fueron los más numerosos, seguido por el Aplicomplexa y en menor medida el pylum Platyhelminthes. Este trabajo menciona por primera vez la presencia de nematodos como Nematodirus sp, Trichuris sp, las coccidias E. macusaniensis, E. ivitaensis y Eimeria sp en guanacos silvestres de la provincia de San Juan. Hubo pocos registros en ambientes con poblaciones pequeñas de guanacos de la ecorregión Puna.
\end{abstract}

Palabras clave: Argentina; endoparásitos; Lama guanicoe; revisión

\section{Abstract}

This study compiles records of endoparasites species in guanacos (Lama guanicoe) from Argentina. A bibliographic review of scientific works published between 1967 and 2017 was conducted. In addition, new unpublished records of localities within the province of San Juan were incorporated. The endoparasite fauna was represented by the phylum Nematoda, Platyhelminthes and Aplicomplexa, with a total of 65 total entries, 26 species

\footnotetext{
${ }^{1}$ Centro de Investigaciones de la Geósfera y la Biosfera (UNSJ-CONICET), Grupo Interacciones Biológicas del Desierto (INTERBIODES), San Juan, Argentina

${ }^{2}$ Universidad Nacional de la Patagonia San Juan Bosco, Sede Puerto Madryn, Chubut, Argentina

${ }^{3}$ E-mail: cynthiajesica.gr@gmail.com
}

Recibido: 7 de mayo de 2018

Aceptado para publicación: 18 de octubre de 2018 
mentioned and 14 others that were only identified at the genus level. The nematodes were the most numerous, followed by the Aplicomplexa and to a lesser extent the pylum Platyhelminthes. This work mentions for the first time the presence of nematodes such as Nematodirus sp, Trichuris sp, coccidia E. macusaniensis, E. ivitaensis and Eimeria sp in wild guanacos from the province of San Juan. There were few records in the environment with small populations of guanacos from the Puna ecoregion.

Key words: Argentina; endoparasites; Lama guanicoe; review

\section{INTRODUCCIÓN}

Los endoparásitos suelen presentar mayor diversidad que los ectoparásitos (Gosling, 2005). Los parásitos que se localizan en el tracto gastrointestinal de los camélidos sudamericanos (CSA) son los más numerosos y los más perjudiciales (Fowler, 2010). Los CSA presentan endoparásitos que son propios de ellos, pero también tienen especies que parasitan animales domésticos con los que comparten áreas de pastoreo o instalaciones para su cría y manejo, especialmente ovejas y cabras (Navone y Merino 1989; Beldomenico et al., 2003; Aguirre y Cafrune, 2007; Petrigh y Fugassa, 2014). Por ello, muchos de los parásitos presentes en los CSA son comunes a las especies domésticas introducidas en los distintos ambientes de América del Sur (De Lamo, 2011).

El guanaco (Lama guanicoe) es el CSA silvestre de mayor tamaño. La mayor abundancia de estos animales en Argentina se observa en la región sur del país (Patagonia), la que se reduce significativamente en las localidades situadas en el centro oeste y noroeste del país (Puig, 1995; Nugent et al., 2006; González y Acebes, 2016). Por ejemplo, en provincias del sur como Chubut y Río Negro se estiman valores que superan ampliamente los $100 \mathrm{mil}$ animales (Gavuzzo et al., 2015) hasta Santa Cruz con una estimación de un millón de ejemplares (González y Acebes, 2016), mientras que en las provincias del noroeste argentino como
Catamarca, Salta y Jujuy se encuentran menos de dos mil individuos (González y Acebes, 2016).

Las amenazas directas que afectan esta variación de abundancias se asignan principalmente a la degradación y fragmentación del hábitat, la caza furtiva, las actividades de comercio ilegal y las interacciones interespecíficas con otros herbívoros. Estos últimos, puede asociarse al contagio de enfermedades, los cuales juegan un rol importante sobre la dinámica de las poblaciones de guanacos (Baldi et al., 2006). Debido a esto, el aporte de los registros parasitarios en este camélido es de gran importancia, tanto para el área veterinaria como para su conservación.

La información sobre endoparásitos en CSA comprende literatura que incluye desde capítulos de libros que describen parásitos de camélidos en general (Aguirre y Cafrune, 2007; Ballweber, 2009) hasta hallazgos por localidad o especie de camélido (Cafrune $e t$ al., 2009a,b; Moreno et al., 2013; entre otros). Sin embargo, esta información no está enfocada exclusivamente a las poblaciones de guanacos de Argentina. Además, existe poca información sanitaria en la zona norte del país, donde las poblaciones son menos abundantes (Cafrune et al., 2009a,b). En este marco, el objetivo de este estudio fue organizar y presentar los registros de endoparásitos de guanacos de Argentina e incorporar los registros de endoparásitos de poblaciones de la provincia de San Juan. 


\section{Materiales y Métodos}

La organización de este trabajo contempló una primera parte de recopilación bibliográfica para la construcción de un cuadro que menciona los registros de endoparásitos en guanacos de Argentina. La segunda parte fue la incorporación de los registros propios de localidades dentro de la provincia de San Juan.

\section{Recopilación Bibliográfica}

Se realizó una búsqueda de la información que hace referencia a endoparásitos obtenidos en muestras de guanaco de Argentina contenida en publicaciones científicas (artículos científicos y capítulos de libros). Solo se utilizaron aquellos trabajos que reportaron endoparásitos identificados a nivel de género o especie. La información se organizó en tres grandes grupos en relación al phylum al cual pertenecieron los parásitos: phylum Apicomplexa (protozoarios), Nematoda (gusanos redondos) y Platyhelminthes (tenias y gusanos chatos).

Para cada género o especie registrada se consignaron los siguientes ítems: nombre científico, provincia/s, localidad/es, número de muestras analizadas (n), prevalencia registrada (\%), estado (situación de cautiverio, semicautiverio o silvestre del animal o grupo de animales examinados) y referencias (fuentes bibliográficas de donde se obtuvo la información).

La recopilación bibliográfica abarcó 50 años (1967-2017), revisándose 16 artículos científicos donde se mencionan registros de endoparásitos en guanacos en Argentina; así como seis publicaciones entre artículos y capítulos de libros relacionadas a aspectos parasitarios en CSA que fueron de consulta general.

\section{Muestreo en San Juan}

En 2014 y 2015 se tomaron muestras de poblaciones de guanacos pertenecientes- a tres áreas protegidas de San Juan, Argentina. Se incluyó la zona de amortiguación de la Reserva Provincial San Guillermo (RSG), con un centro geográfico aproximado de $29^{\circ}$ $3.534^{\prime} \mathrm{S} 69^{\circ} 29.910^{\prime} \mathrm{O}$, la Reserva de uso múltiples Don Carmelo (RDC) con un centro geográfico $31^{\circ} 4.638^{\prime} \mathrm{S}, 69^{\circ} 9.550^{\prime} \mathrm{O}$ y el Parque provincial Ischigualasto (PPI) con un centro geográfico $30^{\circ} 5.096^{\prime} \mathrm{S}, 67^{\circ} 58.997^{\prime}$ O. En las primeras dos reservas se muestreó en localidades pertenecientes a las ecorregiones Puna y Alto Andina y en el PPI en sitios pertenecientes al Monte de Sierras y Bolsones (Pol et al., 2005; Reboratti, 2005).

Para la determinación de fauna parasitaria se tomaron muestras fecales de individuos adultos, lo más frescas posibles. Para ello se procedió a seguir a los grupos de guanacos y, con ayuda de binoculares, se esperó a que defecaran para colectar las muestras (Castillo et al., 2006), identificando la composición del grupo familiar e individuo muestreado.

Cada muestra fue conservada en un envase hermético con formol al 5\% hasta su procesamiento en el Laboratorio de Biología de la Facultad de Ciencias Exactas Físicas y Naturales de la Universidad Nacional de San Juan. Se recolectaron 72 muestras, 33 originarias de RDC, 29 de RSG y 10 de PPI. En el laboratorio se procedió a la identificación de los huevos u ooquistes de las distintas especies de parásitos presentes en la materia fecal. Las muestras se procesaron según la técnica de McMaster modificada (Cafrune et al., 2009a,b).

\section{Resultados y Discusión}

La revisión bibliográfica e incorporación de nuevos registros de endoparásitos de guanacos en Argentina reunió 65 registros, incluyendo 26 parásitos determinados a nivel de especie y otros 14 que solo fueron identificadas a nivel de género (Cuadros 1, 2, 3 y 4). 
Cuadro 1. Registros de especies del phylum Apicomplexa reportados en guanacos de Argentina

\begin{tabular}{|c|c|c|c|c|c|c|}
\hline Género/especie & Provincia & Localidad & N. ${ }^{\circ}$ & $\begin{array}{l}\text { Prevalencia } \\
\text { (n) }\end{array}$ & Estado & Referencia \\
\hline \multirow[t]{6}{*}{ Eimeria $\mathrm{sp}$} & \multirow[t]{2}{*}{ San Juan } & $\begin{array}{l}\text { Rva. Don } \\
\text { Carmelo }\end{array}$ & 33 & 21.2 & Silvestre & $\begin{array}{l}\text { Presente } \\
\text { estudio }\end{array}$ \\
\hline & & $\begin{array}{l}\text { Rva. San } \\
\text { Guillermo }\end{array}$ & 22 & 13.6 & Silvestre & $\begin{array}{l}\text { Presente } \\
\text { estudio }\end{array}$ \\
\hline & \multirow[t]{2}{*}{ Mendoza } & $\begin{array}{l}\text { Rva. La } \\
\text { Payunia }\end{array}$ & 75 & - & Silvestre & $\begin{array}{l}\text { Moreno et al. } \\
\text { (2013) }\end{array}$ \\
\hline & & Malargue & 4 & $24 *$ & $\begin{array}{l}\text { Semi- } \\
\text { cautiverio }\end{array}$ & $\begin{array}{l}\text { Mera y Sierra } \\
\text { et al. }(2015)\end{array}$ \\
\hline & Chubut & $\begin{array}{l}\text { Rva. Cabo } \\
\text { Dos Bahías }\end{array}$ & 12 & $83.3^{*}$ & Silvestre & $\begin{array}{l}\text { Beldomenico et } \\
\text { al. (2003) }\end{array}$ \\
\hline & $\begin{array}{l}\text { Tierra del } \\
\text { Fuego }\end{array}$ & $\begin{array}{l}\text { Península } \\
\text { Mitre }\end{array}$ & 12 & $16.6^{*}$ & Silvestre & $\begin{array}{l}\text { Navone y } \\
\text { Merino (1989) }\end{array}$ \\
\hline E. alpacae & Mendoza & $\begin{array}{l}\text { Rva. La } \\
\text { Payunia }\end{array}$ & 756 & - & Silvestre & $\begin{array}{l}\text { Moreno et al. } \\
(2015)\end{array}$ \\
\hline \multirow[t]{2}{*}{ E. ivitaensis } & San Juan & $\begin{array}{l}\text { Rva. San } \\
\text { Guillermo }\end{array}$ & 22 & 4.5 & Silvestre & $\begin{array}{l}\text { Presente } \\
\text { estudio }\end{array}$ \\
\hline & Mendoza & $\begin{array}{l}\text { Rva. La } \\
\text { Payunia }\end{array}$ & 756 & - & Silvestre & $\begin{array}{l}\text { Moreno et al. } \\
(2015)\end{array}$ \\
\hline \multirow[t]{5}{*}{$\begin{array}{l}\text { E. } \\
\text { macusaniensis }\end{array}$} & Salta & $\begin{array}{l}\text { No } \\
\text { reportado }\end{array}$ & 4 & 25 & $\begin{array}{l}\text { No } \\
\text { reportado }\end{array}$ & $\begin{array}{l}\text { Cafrune et al. } \\
(2009 a)\end{array}$ \\
\hline & \multirow[t]{2}{*}{ San Juan } & $\begin{array}{l}\text { Rva. Don } \\
\text { Carmelo }\end{array}$ & 33 & 36.3 & Silvestre & $\begin{array}{l}\text { Presente } \\
\text { estudio }\end{array}$ \\
\hline & & $\begin{array}{l}\text { Rva. San } \\
\text { Guillermo }\end{array}$ & 22 & 36 & Silvestre & $\begin{array}{l}\text { Presente } \\
\text { estudio }\end{array}$ \\
\hline & Mendoza & $\begin{array}{l}\text { Rva. La } \\
\text { Payunia }\end{array}$ & $75 ; 756$ & - & Silvestre & $\begin{array}{l}\text { Moreno et al. } \\
\text { (2013); Moreno } \\
\text { et al. }(2015)\end{array}$ \\
\hline & Chubut & $\begin{array}{l}\text { Rva. Cabo } \\
\text { Dos Bahías }\end{array}$ & 12 & $75^{*}$ & Silvestre & $\begin{array}{l}\text { Beldomenico et } \\
\text { al. (2003) }\end{array}$ \\
\hline E. lamae & Mendoza & $\begin{array}{l}\text { Rva. La } \\
\text { Payunia }\end{array}$ & 756 & - & Silvestre & $\begin{array}{l}\text { Moreno et al. } \\
(2015)\end{array}$ \\
\hline E. punoensis & Mendoza & $\begin{array}{l}\text { Rva. La } \\
\text { Payunia }\end{array}$ & 756 & - & Silvestre & $\begin{array}{l}\text { Moreno et al. } \\
(2015)\end{array}$ \\
\hline Sarcocitys sp & Chubut & $\begin{array}{l}\text { Rva. Cabo } \\
\text { Dos Bahías }\end{array}$ & 12 & $66.6^{*}$ & Silvestre & $\begin{array}{l}\text { Beldomenico et } \\
\text { al. (2003) }\end{array}$ \\
\hline \multirow[t]{2}{*}{ S. aucheniae } & $\begin{array}{l}\text { Santa } \\
\text { Cruz }\end{array}$ & $\begin{array}{l}\text { No } \\
\text { reportado }\end{array}$ & 2 & 100 & $\begin{array}{l}\text { No } \\
\text { reportado }\end{array}$ & $\begin{array}{l}\text { Regensburger } \\
\text { et al. }(2015)\end{array}$ \\
\hline & $\begin{array}{l}\text { Santa } \\
\text { Cruz }\end{array}$ & $\begin{array}{l}\text { No } \\
\text { reportado }\end{array}$ & - & - & $\begin{array}{l}\text { No } \\
\text { reportado }\end{array}$ & $\begin{array}{l}\text { Moré et al. } \\
(2016)\end{array}$ \\
\hline S. mansoni & $\begin{array}{l}\text { Santa } \\
\text { Cruz }\end{array}$ & $\begin{array}{l}\text { No } \\
\text { reportado }\end{array}$ & - & - & $\begin{array}{l}\text { No } \\
\text { reportado }\end{array}$ & $\begin{array}{l}\text { Moré et al. } \\
(2016)\end{array}$ \\
\hline S. tilopodi & Mendoza & $\begin{array}{l}\text { Co. El } \\
\text { Nevado y } \\
\text { co. Payún }\end{array}$ & 119 & 41.3 & Silvestre & $\begin{array}{l}\text { Quiroga y } \\
\text { Lombardero } \\
(1967)\end{array}$ \\
\hline
\end{tabular}

* Prevalencia calculada con los datos publicados 
Cuadro 2. Registros de especies del phylum Nematoda reportados en guanacos de Argentina. Parte I

\begin{tabular}{|c|c|c|c|c|c|c|}
\hline Género / especie & Provincia & Localidad & N. ${ }^{\circ}$ & $\begin{array}{l}\text { Prevalencia } \\
\text { (n) }\end{array}$ & Estado & Referencia \\
\hline \multirow[t]{2}{*}{ Capillaria $\mathrm{sp}$} & Río Negro & $\begin{array}{l}\text { No } \\
\text { reportado }\end{array}$ & 3 & $66.6^{*}$ & $\begin{array}{l}\text { No } \\
\text { reportado }\end{array}$ & $\begin{array}{l}\text { Larrieu et al. } \\
\text { (1982) }\end{array}$ \\
\hline & Mendoza & $\begin{array}{l}\text { Rva. La } \\
\text { Payunia }\end{array}$ & 756 & - & Silvestre & $\begin{array}{l}\text { Moreno et al. } \\
(2015)\end{array}$ \\
\hline Chabertia sp & $\begin{array}{l}\text { Tierra del } \\
\text { Fuego }\end{array}$ & $\begin{array}{l}\text { Península } \\
\text { Mitre }\end{array}$ & - & - & Silvestre & $\begin{array}{l}\text { Navone y } \\
\text { Merino (1989) }\end{array}$ \\
\hline Cooperia sp & $\begin{array}{l}\text { Tierra del } \\
\text { Fuego }\end{array}$ & $\begin{array}{l}\text { Península } \\
\text { Mitre }\end{array}$ & - & - & Silvestre & $\begin{array}{l}\text { Navone y } \\
\text { Merino (1989) }\end{array}$ \\
\hline $\begin{array}{l}\text { Cooperia } \\
\text { oncophora }\end{array}$ & Río Negro & $\begin{array}{l}\text { No } \\
\text { reportado }\end{array}$ & 3 & $33.3 *$ & $\begin{array}{l}\text { No } \\
\text { reportado }\end{array}$ & $\begin{array}{l}\text { Larrieu et al. } \\
\text { (1982) }\end{array}$ \\
\hline $\begin{array}{l}\text { Cooperia } \\
\text { mcmasteri }\end{array}$ & Río Negro & $\begin{array}{l}\text { No } \\
\text { reportado }\end{array}$ & 3 & $100 *$ & $\begin{array}{l}\text { No } \\
\text { reportado }\end{array}$ & $\begin{array}{l}\text { Larrieu et al. } \\
\text { (1982) }\end{array}$ \\
\hline Dictyocaulus sp & Chubut & $\begin{array}{l}\text { Rva. Cabo } \\
\text { Dos Bahías }\end{array}$ & 12 & $8.3^{*}$ & Silvestre & $\begin{array}{l}\text { Karesh et al. } \\
\text { (1998) }\end{array}$ \\
\hline $\begin{array}{l}\text { Dictyocaulus } \\
\text { filaria }\end{array}$ & Chubut & $\begin{array}{l}\text { Rva. Cabo } \\
\text { Dos Bahías }\end{array}$ & 12 & $83.3 *$ & Silvestre & $\begin{array}{l}\text { Beldomenico et } \\
\text { al. (2003) }\end{array}$ \\
\hline $\begin{array}{l}\text { Dictyocaulus } \\
\text { filaria }\end{array}$ & Río Negro & Bariloche & - & - & Cautiverio & $\begin{array}{l}\text { Sarasqueta } \\
(2001)\end{array}$ \\
\hline Haemonchus sp & $\begin{array}{l}\text { Tierra del } \\
\text { Fuego }\end{array}$ & $\begin{array}{l}\text { Península } \\
\text { Mitre }\end{array}$ & - & - & Silvestre & $\begin{array}{l}\text { Navone y } \\
\text { Merino (1989) }\end{array}$ \\
\hline $\begin{array}{l}\text { Lamanema } \\
\text { chevazi }\end{array}$ & Salta & Cachi & 4 & 75 & $\begin{array}{l}\text { No } \\
\text { reportado }\end{array}$ & $\begin{array}{l}\text { Cafrune et al. } \\
(2009 b)\end{array}$ \\
\hline \multirow[t]{2}{*}{ Marshallagia sp } & Chubut & $\begin{array}{l}\text { Rva. Cabo } \\
\text { Dos Bahías }\end{array}$ & $\begin{array}{l}12 ; \\
12\end{array}$ & - & Silvestre & $\begin{array}{l}\text { Karesh et al. } \\
(1998) ; \\
\text { Beldomenico et } \\
\text { al. (2003) }\end{array}$ \\
\hline & $\begin{array}{l}\text { Tierra del } \\
\text { Fuego }\end{array}$ & $\begin{array}{l}\text { Península } \\
\text { Mitre }\end{array}$ & - & - & Silvestre & $\begin{array}{l}\text { Navone y } \\
\text { Merino (1989) }\end{array}$ \\
\hline \multirow[t]{4}{*}{ Nematodirus sp } & San Juan & $\begin{array}{l}\text { Rva. Don } \\
\text { Carmelo }\end{array}$ & 33 & 15.1 & Silvestre & $\begin{array}{l}\text { Presente } \\
\text { estudio }\end{array}$ \\
\hline & Mendoza & $\begin{array}{l}\text { Rva. La } \\
\text { Payunia }\end{array}$ & 75 & & Silvestre & $\begin{array}{l}\text { Moreno et al. } \\
\text { (2013) }\end{array}$ \\
\hline & Chubut & $\begin{array}{l}\text { Rva. Cabo } \\
\text { Dos Bahías }\end{array}$ & $\begin{array}{l}12 \\
12\end{array}$ & $50 * ; 75^{*}$ & Silvestre & $\begin{array}{l}\text { Karesh et al. } \\
(1998) ; \\
\text { Beldomenico et } \\
\text { al. (2003) }\end{array}$ \\
\hline & $\begin{array}{l}\text { Tierra del } \\
\text { Fuego }\end{array}$ & $\begin{array}{l}\text { Península } \\
\text { Mitre }\end{array}$ & - & - & Silvestre & $\begin{array}{l}\text { Navone y } \\
\text { Merino (1989) }\end{array}$ \\
\hline \multirow[t]{3}{*}{ N. spathiger } & Mendoza & $\begin{array}{l}\text { Rva. La } \\
\text { Payunia }\end{array}$ & 756 & & $\begin{array}{l}\text { Silvestre } \\
\text { Moreno et } \\
\text { al. }(2013)\end{array}$ & $\begin{array}{l}\text { Moreno et al. } \\
(2015)\end{array}$ \\
\hline & Río Negro & $\begin{array}{l}\text { No } \\
\text { reportado }\end{array}$ & 3 & $33.3 *$ & $\begin{array}{l}\text { No } \\
\text { reportado }\end{array}$ & $\begin{array}{l}\text { Larrieu et al. } \\
\text { (1982) }\end{array}$ \\
\hline & Santa Cruz & $\begin{array}{l}\text { Pque. Nac. } \\
\text { Perito } \\
\text { Moreno }\end{array}$ & - & - & Silvestre & $\begin{array}{l}\text { Petrigh y } \\
\text { Fugassa (2014) }\end{array}$ \\
\hline
\end{tabular}

* Prevalencia calculada con los datos publicados 
Cuadro 3. Registros de especies del phylum Nematoda reportados en guanacos de Argentina. Parte II

\begin{tabular}{|c|c|c|c|c|c|c|}
\hline $\begin{array}{l}\text { Género } \\
\text { /especie }\end{array}$ & Provincia & Localidad & N. ${ }^{\circ}$ & $\begin{array}{l}\text { Prevalencia } \\
\text { (n) }\end{array}$ & Estado & Referencia \\
\hline N. helvetianus & Mendoza & $\begin{array}{l}\text { Rva. La } \\
\text { Payunia }\end{array}$ & 756 & - & Silvestre & $\begin{array}{l}\text { Moreno et al. } \\
\text { (2015) }\end{array}$ \\
\hline N. lanceolatus & Río Negro & $\begin{array}{l}\text { No } \\
\text { reportado }\end{array}$ & 3 & $33.3^{*}$ & $\begin{array}{l}\text { No } \\
\text { reportado }\end{array}$ & $\begin{array}{l}\text { Larrieu et al. } \\
\text { (1982) }\end{array}$ \\
\hline N. fillicolis & Río Negro & $\begin{array}{l}\text { No } \\
\text { reportado }\end{array}$ & 3 & $33.3^{*}$ & $\begin{array}{l}\text { No } \\
\text { reportado }\end{array}$ & $\begin{array}{l}\text { Larrieu et al. } \\
\text { (1982) }\end{array}$ \\
\hline N. battus & Río Negro & $\begin{array}{l}\text { No } \\
\text { reportado }\end{array}$ & 3 & $33.3^{*}$ & $\begin{array}{l}\text { No } \\
\text { reportado }\end{array}$ & $\begin{array}{l}\text { Larrieu et al. } \\
\text { (1982) }\end{array}$ \\
\hline $\begin{array}{l}\text { Oesofagostomun } \\
\text { sp }\end{array}$ & $\begin{array}{l}\text { Tierra del } \\
\text { Fuego }\end{array}$ & $\begin{array}{l}\text { Península } \\
\text { Mitre }\end{array}$ & - & - & Silvestre & $\begin{array}{l}\text { Navone y } \\
\text { Merino (1989) }\end{array}$ \\
\hline Ostertagia sp & $\begin{array}{l}\text { Tierra del } \\
\text { Fuego }\end{array}$ & $\begin{array}{l}\text { Península } \\
\text { Mitre }\end{array}$ & - & - & Silvestre & $\begin{array}{l}\text { Navone y } \\
\text { Merino (1989) }\end{array}$ \\
\hline O. ostertagi & Río Negro & $\begin{array}{l}\text { No } \\
\text { reportado }\end{array}$ & 3 & $100^{*}$ & $\begin{array}{l}\text { No } \\
\text { reportado }\end{array}$ & $\begin{array}{l}\text { Larrieu et al. } \\
\text { (1982) }\end{array}$ \\
\hline \multirow[t]{2}{*}{$\begin{array}{l}\text { Trichostrongylus } \\
\text { sp }\end{array}$} & Chubut & $\begin{array}{l}\text { Rva. Cabo } \\
\text { Dos } \\
\text { Bahías }\end{array}$ & 12 & $25^{*}$ & Silvestre & $\begin{array}{l}\text { Karesh et al. } \\
\text { (1998) }\end{array}$ \\
\hline & $\begin{array}{l}\text { Tierra del } \\
\text { fuego }\end{array}$ & $\begin{array}{l}\text { Península } \\
\text { Mitre }\end{array}$ & - & - & Silvestre & $\begin{array}{l}\text { Navone y } \\
\text { Merino (1989) }\end{array}$ \\
\hline T. axei & Río Negro & $\begin{array}{l}\text { No } \\
\text { reportado }\end{array}$ & 3 & $100^{*}$ & $\begin{array}{l}\text { No } \\
\text { reportado }\end{array}$ & $\begin{array}{l}\text { Larrieu et al. } \\
\text { (1982) }\end{array}$ \\
\hline T. vitrinus & Río Negro & $\begin{array}{l}\text { No } \\
\text { reportado }\end{array}$ & 3 & $66.6^{*}$ & $\begin{array}{l}\text { No } \\
\text { reportado }\end{array}$ & $\begin{array}{l}\text { Larrieu et al. } \\
\text { (1982) }\end{array}$ \\
\hline \multirow[t]{4}{*}{ Trichuris sp } & San Juan & $\begin{array}{l}\text { Rva. Don } \\
\text { Carmelo }\end{array}$ & 33 & 15.1 & Silvestre & $\begin{array}{l}\text { Presente } \\
\text { estudio }\end{array}$ \\
\hline & & $\begin{array}{l}\text { Rva. San } \\
\text { Guillermo }\end{array}$ & 22 & 4.5 & Silvestre & $\begin{array}{l}\text { Presente } \\
\text { estudio }\end{array}$ \\
\hline & Mendoza & $\begin{array}{l}\text { Rva. La } \\
\text { Payunia }\end{array}$ & 756 & & Silvestre & $\begin{array}{l}\text { Moreno et al. } \\
\text { (2015) }\end{array}$ \\
\hline & Chubut & $\begin{array}{l}\text { Rva. Cabo } \\
\text { Dos } \\
\text { Bahías }\end{array}$ & 12 & $41.6^{*}$ & Silvestre & $\begin{array}{l}\text { Karesh et al. } \\
\text { (1998) }\end{array}$ \\
\hline T. ovis & Río Negro & $\begin{array}{l}\text { No } \\
\text { reportado }\end{array}$ & 3 & $33.3^{*}$ & $\begin{array}{l}\text { No } \\
\text { reportado }\end{array}$ & $\begin{array}{l}\text { Larrieu et al. } \\
\text { (1982) }\end{array}$ \\
\hline T. tenuis & Chubut & $\begin{array}{l}\text { Rva. Cabo } \\
\text { Dos } \\
\text { Bahías }\end{array}$ & 12 & $41.6^{*}$ & Silvestre & $\begin{array}{l}\text { Beldomenico } \\
\text { et al. (2003) }\end{array}$ \\
\hline Skrjabinema ovis & Río Negro & $\begin{array}{l}\text { No } \\
\text { reportado }\end{array}$ & 3 & $33.3^{*}$ & $\begin{array}{l}\text { No } \\
\text { reportado }\end{array}$ & $\begin{array}{l}\text { Larrieu et al., } \\
\text { (1982) }\end{array}$ \\
\hline \multirow[t]{2}{*}{ Strongyloides sp } & Chubut & $\begin{array}{l}\text { Rva. Cabo } \\
\text { Dos } \\
\text { Bahías }\end{array}$ & 12 & $8.3^{*}$ & Silvestre & $\begin{array}{l}\text { Karesh et al. } \\
\text { (1998) }\end{array}$ \\
\hline & Mendoza & $\begin{array}{l}\text { Rva. La } \\
\text { Payunia }\end{array}$ & 756 & - & Silvestre & $\begin{array}{l}\text { Moreno et al. } \\
\text { (2015) }\end{array}$ \\
\hline
\end{tabular}

* Prevalencia calculada con los datos publicados 
Cuadro 4. Registros de especies de los phylum Platyhelminthes (Cestoda y Trematoda) reportados en guanacos de Argentina

\begin{tabular}{|c|c|c|c|c|c|c|}
\hline $\begin{array}{l}\text { Género/ } \\
\text { especie }\end{array}$ & Provincia & Localidad & $\mathrm{N} .^{\circ}$ & $\begin{array}{l}\text { Prevalencia } \\
\text { (n) }\end{array}$ & Estado & Referencia \\
\hline $\begin{array}{l}\text { Moniezia } \\
\text { benedeni }\end{array}$ & Mendoza & $\begin{array}{l}\text { Rva. La } \\
\text { Payunia }\end{array}$ & 756 & - & Silvestre & $\begin{array}{l}\text { Moreno et al. } \\
\text { (2015) }\end{array}$ \\
\hline $\begin{array}{l}\text { Moniezia } \\
\text { expansa }\end{array}$ & Chubut & $\begin{array}{l}\text { Rva. Cabo } \\
\text { Dos Bahías }\end{array}$ & 12 & $16.6^{*}$ & Silvestre & $\begin{array}{l}\text { Beldomenico et } \\
\text { al. (2003) }\end{array}$ \\
\hline \multirow[t]{3}{*}{$\begin{array}{l}\text { Facsiola } \\
\text { hepática }\end{array}$} & Mendoza & $\begin{array}{l}\text { Rva. La } \\
\text { Payunia y } \\
\text { Laguna de } \\
\text { Llancanelo }\end{array}$ & 224 & 0.5 & Silvestre & Issia et al. (2009) \\
\hline & & Malargüe & 4 & $25^{*}$ & $\begin{array}{l}\text { Semi- } \\
\text { cautiverio }\end{array}$ & $\begin{array}{l}\text { Mera y Sierra et } \\
\text { al. }(2015)\end{array}$ \\
\hline & $\begin{array}{l}\text { Río } \\
\text { Negro }\end{array}$ & $\begin{array}{l}\text { No } \\
\text { reportado }\end{array}$ & - & - & $\begin{array}{l}\text { Semi- } \\
\text { cautiverio }\end{array}$ & Olaechea (2007) \\
\hline
\end{tabular}

* Prevalencia calculada con los datos publicados

Se registraron dos géneros del grupo de protozoarios, cinco especies del género Eimeria y tres del género Sarcocistys (Cuadro 1). Para el phylum Nematoda se mencionan dos especies del género Cooperia, una de Dictyocaulus, una de Lamanema, cinco de Nematodirus, uno de Ostertagia, dos de Trichostrongylus, dos Trichuris y una Skrjabinema (Cuadros 2 y 3). Para los cestodos se encontraron dos registros de especies del género Moniezia, y solo una especie de trematodo, la Fasciola hepatica (Cuadro 4).

El número de especies de parásitos con relación a las ecorregiones muestra que las poblaciones de la estepa patagónica representan el mayor registro de riqueza (Figura 1), seguida por el Bosque Patagónico y en menor medida la Puna, con seis taxa que representan los registros de la provincia de Salta y las incorporadas en este trabajo. De esta manera, este estudio representa el segundo trabajo de diagnóstico de endoparásitos realizado con guanacos de la Puna. Por otro lado, con relación al estado de cautividad de los guanacos, se encuentra que el mayor núme- ro de registros está relacionado a poblaciones de animales silvestres (Figura 2).

Entre las coccidias, existen especies de los géneros Eimeria y Sarcocistys que parasitan exclusivamente a los CSA, de las cuales están mencionadas cinco de las seis especies descritas de Eimeria y tres de las cuatro especies citadas del género Sarcocistys.

Para el grupo de nematodos, Lamanema chavezi (Cafrune et al., 2009b) y Trichuris tenuis (Beldomenico et al., 2003) son las especies específicas de CSA mencionadas en Argentina. Con respecto a los géneros Capillaria y Nematodirus, se incluyen especies exclusivas de CSA y otras compartidas, pero no han sido identificadas las especies.

Para el trematodo Fasciola hepatica, los registros son más escasos siendo el primer registro en semicautividad el de Olaechea (2007) y el primero en silvestres el de Issia et al. (2009). Para los cestodos, no existen representantes actuales de Moniezia en CSA 


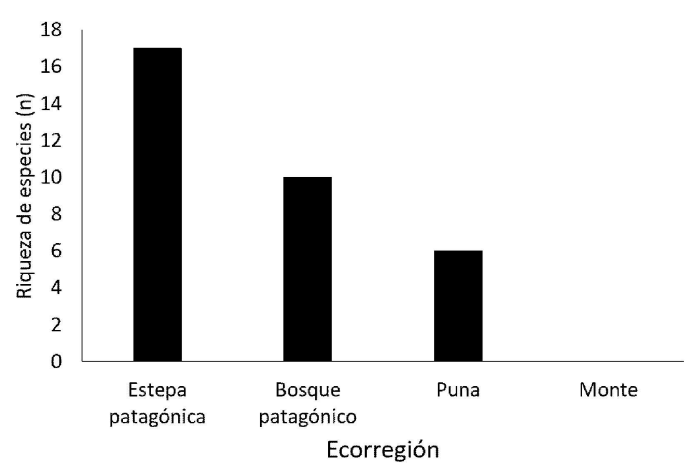

Figura 1. Número de especies de endoparásitos en guanacos en función a la ecorregión de los registros según las localidades reportadas

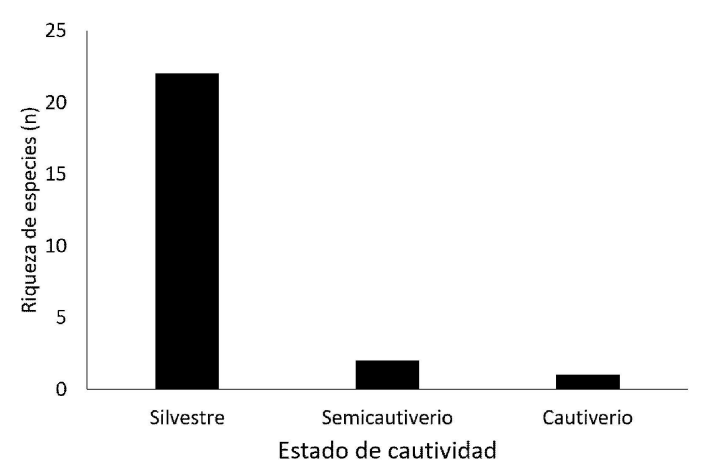

Figura 2. Número de especies de endoparásitos en guanacos en función al estado de cautividad de los ejemplares reportado

(Denegri, 2001), de allí que los registros mencionados por Beldomenico et al. (2003) y Moreno et al. (2015) pertenecen a zonas donde posiblemente el rumiante doméstico comparte territorio con el guanaco.

La provincia de Mendoza es la única que registra representantes de todos los phylum, seguida por Chubut con tres de los cuatro grupos. El resto de las provincias solo mencionan especies del grupo nematoda $y / o$ aplicompexa. En este trabajo se menciona por primera vez para San Juan la presencia de nematodos como Nematodirus sp, Trichuris sp, las coccidias E. macusaniensis, E. ivitaensis y Eimeria spp en guanacos silvestres. Trichuris sp, E. macusaniensis y Nematodirus sp fueron detectados en PSG y RDC, Nematodirus sp y Eimeria spp solo en RDC, E. ivitaensis solo en PSG. Por otro lado, no se encontraron endoparásitos en la población de PPI. La no detección de endoparásitos en el PPI podría ser debido a que la abundancia o prevalencia de estos parásitos en esta localidad sea muy baja para poder haber sido detectada en este estudio.

Las provincias que presentaron mayor prevalencia fueron las de Río Negro, Chubut y Santa Cruz y Salta. En el caso del protozoo Sarcosistys aucheniae se encontró en los dos animales estudiados en Santa Cruz (Regensburger et al., 2015). En el caso de los nematodes Cooperia mcmasteri, $O$. ostertagi y $T$. axei, su prevalencia fue del $100 \%$ sobre tres animales de Río Negro (Larrieu et al. 1982).

Teniendo en cuenta las estimaciones poblacionales, las provincias con valores que superan ampliamente los 100 mil animales como Chubut, Río Negro, Santa Cruz, detectaron entre todas a 21 especies de endoparásitos. Por otro lado, las provincias con estimaciones entre 10 y 20 mil animales como Tierra del Fuego, Mendoza y San Juan registraron en forma conjunta a 20 especies, pero esto incluye el sur de Mendoza, donde ya comienza la Patagonia. Entre las que tienen menos de dos mil animales como Jujuy, Catamarca, La Rioja, Salta, La Pampa, Córdoba y San Luis, solo existen dos registros para Jujuy. En Neuquén tampoco existen registros de endoparásitos de guanaco, a pesar de tener una población de guanacos estimada en más de 30 mil ejemplares.

En resumen, las poblaciones con más información están relacionadas a poblaciones de mayor tamaño, de la región patagónica 
y de animales en estado silvestre. Esto muestra que si bien existen una variedad de registros de endoparásitos, los resultados del presente estudio aportan información valiosa sobre las especies parásitas de poblaciones de guanacos silvestres de mediana abundancia del extremo sur árido de la ecorregión de Puna.

\section{Conclusiones}

- Se han identificado 26 especies de endoparásitos en guanacos en Argentina y otras 14 a nivel de género.

- Se incorporan nuevos registros de endoparásitos en guanacos silvestres de la provincia de San Juan (Nematodirus sp, Trichuris sp y las coccidias E. macusaniensis, E. ivitaensis y Eimeria spp.

\section{Literatura Citada}

1. Aguirre DH, Cafrune MM. 2007. Parásitos de los camélidos sudamericanos. En: Suárez VH, Olaechea FV, Romero JR, Rossanigo CE (eds). Enfermedades parasitarias de los ovinos y otros rumiantes menores del Cono Sur. Argentina: INTA. p 141-147.

2. Baldi R, De Lamo D, Failla M, Ferrando P, Funes M, Nugent P, Puig $S$, et al. 2006. Plan nacional de manejo del guanaco (Lama guanicoe). República Argentina. Secretaría de Ambiente y Desarrollo Sustentable de la Nación. 57 p.

3. Ballweber LR. 2009. Ecto-and endoparasites of new world camelids. Vet Clin N Am-Food A 25: 295-310. doi: 10.1016/j.cvfa.2009.02.003

4. Beldomenico PM, Uhart M, Bono MF, Marull C, Baldi R, Peralta JL. 2003. Internal parasites of free-ranging guanacos from Patagonia. Vet Parasitol 118: 71-77. doi: 10.1016/j.vetpar.-2003.09.008
5. Cafrune MM, Marín RE, Rigalt FA, Romero SR, Aguirre DH. 2009 a. Lamanema chavezi (Nematoda: Molineidae): epidemiological data of the infection in South American camelids of Northwest Argentina. Vet Parasitol 166: 321-325. doi: 10.1016/j.vetpar.2009.09.008

6. Cafrune MM, Marín RE, Rigalt FA, Romero SR, Aguirre DH. 2009 b. Prevalence of Eimeria macusaniensis and Eimeria ivitaensis in South American camelids of Northwest Argentina. Vet Parasitol 162: 338-341. doi: 10.1016/ j.vetpar.2009.03.006

7. Castillo D, Chávez V, Hoces R, Casas A, Rosadio A, Wheeler JC. 2008. Contribución al estudio del parasitismo gastrointestinal en guanacos (Lama guanicoe cacsilensis). Rev Inv Vet Peru 19: 168-175. doi: 10.15381/ rivep.v19i2.1164

8. De Lamo D. 2011. Camélidos sudamericanos. Historia, usos y sanidad animal. SENASA 37 p. [Internet]. Disponible en: http://www.vetcomunicaciones.com.ar/ uploadsarchivos/cam_lidos_sudamericanos.pdf

9. Denegri GM. 2001. Cestodosis de herbívoros domésticos de la República Argentina de importancia en medicina veterinaria. Mar del Plata, Argentina: Ed Martín. $111 \mathrm{p}$.

10. Fowler ME. 2010. Medicine and surgery of camelids. $3^{\text {ed }}$. Iowa: WileyBlackwell. $644 \mathrm{p}$.

11. Gavuzzo AB, Gáspero P, Bernardos J, Pedrana J, De Lamo D, Von Thüngen J. 2015. Distribución y densidad de guanacos (Lama guanicoe) en la Patagonia: informe relevamiento 20142015. Bariloche, Río Negro: INTA. 39 p. [Internet]. Disponible en: https://inta.gob.ar/sites/default/files/informe_relevamiento_guanacos_abg_el_al_20132014.pdf 
12. González BA, Acebes P. 2016. Reevaluación del guanaco para la Lista Roja de la UICN: situación actual y recomendaciones a futuro. GECS News 6: 15-20.

13. Gosling PJ. 2005. Dictionary of parasitology. Boca Raton, USA: CRC. 408 p.

14. Issia L, Pietrokovsky S, SousaFigueiredo J, Stothard JR, Wisnivesky-Colli C. 2009. Fasciola hepa-tica infections in livestock flock, guanacos and coypus in two wildlife reserves in Argentina. Vet Parasitol 165: 341-344. doi: 10.1016/j.vetpar.-2009.07.011

15. Karesh W, Uhart M, Dierenfeld E, Braselton W, Torres A, House C, Puche H, et al. 1998. Health evaluation of free-ranging guanaco (Lama guanicoe). J Zoo Wildlife Med 29: 134-141.

16. Larrieu E, Bigatti R, Lukovich R, Eddi C, Banazzi E, Gómez E, Niec R, et al. 1982. Contribución al estudio del parasitismo gastrointestinal en guanacos (Lama guanicoe) y llamas (Lama glama). Gaceta Vet 44: 958-960.

17. Mera y Sierra R, Cantero F, González M. 2015. Fasciola hepatica en guanacos y llamas en un establecimiento de Malargüe, provincia de Mendoza. Rev Argentina Zoonosis Enf Infec Emerg 10: 46-47.

18. Moré G, Regensburger C, Gos ML, Pardini L, Verma SK, Ctibor J, SerraMartinez ME, et al. 2016. Sarcocystis masoni, n. sp. (Apicomplexa: Sarcocystidae), and redescription of Sarcocystis aucheniae from llama (Lama glama), guanaco (Lama guanicoe) and alpaca (Vicugna pacos). Parasitology 143: 617-626. doi: 10.1017/S00311$8201600007 \mathrm{X}$

19. Moreno PG, Eberhardt MAT, Lamattina D, Previtali MA, Beldomenico PM. 2013. Intra-phylum and interphyla associations among gastrointestinal parasites in two wild mammal species. Parasitol Res 112: 3295-3304.
20. Moreno PG, Schroeder NM, Taraborelli P, Gregorio P, Carmanchahi PD, Beldomenico PM. 2015. La comunidad de parásitos gastrointestinales de guanacos silvestres (Lama guanicoe) de la reserva provincial La Payunia, Mendoza, Argentina. Mastozoología Neotropical 22: 63-71.

21. Navone G, Merino M. 1989. Contribución al conocimiento de la fauna endoparasitaria de Lama guanicoe, Muller, 1776, de Península Mitre, Tierra del Fuego, Argentina. Bol Chil Parasitol 44: 46-51.

22. Nugent P, Baldi R, Carmanchahi P, De Lamo D, Failla M, Ferrando $P$, Funes M, et al. 2006. Conservación del guanaco en la Argentina. Propuesta para un plan nacional de manejo. En: Bolkovic ML, Ramadori D (eds). Manejo de fauna silvestre en la Argentina. Programas de uso sustentable. Buenos Aires: Ministerio de Salud y Ambiente de la Nación. p 137-150.

23. Olaechea F. 2007. Fasciola hepatica. En: Suárez VH, Olaechea FV, Romero JR, Rossanigo CE, (eds). Enfermedades parasitarias de los ovinos y otros rumiantes menores del Cono Sur. Argentina: INTA. p 159-168.

24. Petrigh RS, Fugassa MH. 2014. Molecular identification of Nematodirus spathiger (Nematoda: Molineidae) in Lama guanicoe from Patagonia, Argentina. Helminthologia 51: 79-82. doi: 10.2478/s11687-014-0213-z

25. Pol RG, Camín SR, Astié AA. 2005. Situación ambiental en la ecorregión del monte. En: Brown A, Martinez Ortiz U, Acerbi M, Corcuera JF (eds). La situación ambiental Argentina. Buenos Aires: Fundación Vida Silvestre Argentina. p 227-233.

26. Puig S. 1995. Abundancia y distribución de las poblaciones de guanaco. En: Puig $\mathrm{S}$ (ed). Técnicas para el manejo del guanaco. Gland, Suiza: UICN. p 57-70.

27. Quiroga D, Lombardero O, Zorrilla R. 1969. Sarcocystis tilopodi n sp. en- 
guanacos (Lama guanicoe) de la República Argentina. Gaceta Vet 31: 67-70.

28. Reboratti C. 2005. Situación ambiental en las ecorregiones Puna y Altos Andes. En: Brown A, Martinez Ortiz U, Acerbi M, Corcuera JF (eds). La situación ambiental Argentina 2005. Buenos Aires: Fundación Vida Silvestre Argentina. $p$ 33-51.
29. Regensburger C, Gos ML, Ctibor J, Moré G .2015. Morphological and molecular characteristics of Sarcocystis aucheniae isolated from meat of guanaco (Lama guanicoe). J Food Quality Hazards Control 2: 118-121.

30. Sarasqueta DV. 2001. Cría y reproducción de guanacos en cautividad (Lama guanicoe). Comunicación técnica $\mathrm{N}^{\mathrm{O}}$ 110. Argentina: INTA Bariloche. 78 p. 